Supporting Information for "A simple ethanol refluxing method for production of blue colored titanium dioxide with oxygen vacancies and visible light-driven photocatalytic properties".

Stefano Lettieri, ${ }^{1 *}$, Valentina Gargiulo ${ }^{2}$, Michela Alfè ${ }^{2 *}$, Matteo Amati ${ }^{3}$, Patrick Zeller, ${ }^{3}$ ValentinAdrian Maraloiu, ${ }^{4}$ Fabio Borbone, ${ }^{5}$ Michele Pavone, ${ }^{5}$ Ana B. Muñoz-García, ${ }^{6}$ Pasquale Maddalena ${ }^{6}$

${ }^{1}$ Institute of Applied Sciences and Intelligent Systems, National Research Council (ISASI-CNR), Via Campi Flegrei 34, 80078 Pozzuoli (NA), Italy.

${ }^{2}$ Institute for Research on Combustion, National Research Council (IRC-CNR), Piazzale V. Tecchio 80, 80125 Napoli, Italy.

${ }^{3}$ Elettra Sincrotrone Trieste SCPA di interesse nazionale, SS14 Km 163-5, I-34149 Trieste, Italy

${ }^{4}$ National Institute of Materials Physics, 405 Atomistilor Str, 077125 Magurele, Romania.

${ }_{5}^{5}$ Dipartimento di Scienze Chimiche, Università degli Studi di Napoli "Federico II", Complesso Universitario di Monte S. Angelo, Via Cintia, 80126 Napoli, Italy

${ }^{6}$ Dipartimento di Fisica, Università degli Studi di Napoli "Federico II", Complesso Universitario di Monte S. Angelo, Via Cintia, 80126 Napoli, Italy.

\title{
List of figures:
}

Figure S1 Pictures of P25 powder immediately before (left column) and after (center and right columns) a 24 hours of alcohol refluxing treatments at both the explored temperatures in three alcohols. Center column: oil bath temperature equal to alcohol boiling temperature plus $10^{\circ} \mathrm{C}$. Right column: oil bath temperature equal to $120^{\circ} \mathrm{C}$.

Figure S2 Comparison among the $F(\lambda)$ curves and the pictures of P25, P25EtOH(88) and P25EtOH.

Figure S3 Comparison among the $\mathrm{F}(\lambda)$ curves and the pictures of Anat, AnatEtOH, AnatMeOH and AnatPrOH.

Figure S4 XRD spectra of P25 and P25EtOH samples.

Figure S5 Histogram of particle size measured via HRTEM for pristine P25 and P25EtOH samples.

Figure S6 SPEM-XPS survey plot for P25EtOH-24 nanoparticles.

Figure S7 Map of Ti $2 p$ and 0 1s signal from P25EtOH nanoparticles on a 50x50 $\mu^{2}$ area, obtained using the SPEM-XPS in microscopy imaging mode.

Figure S8 Projected density of states calculated for bulk anatase and for stoichiometric (101) anatase.

Figure S9 Spectral intensity of the light source employed for the MB photocatalytic tests.

Figure S10 Absorption spectra of $\mathrm{MB}$ aqueous solution in absence of $\mathrm{TiO}_{2}$ photocatalyst before and after 150 minutes of VIS illumination. 

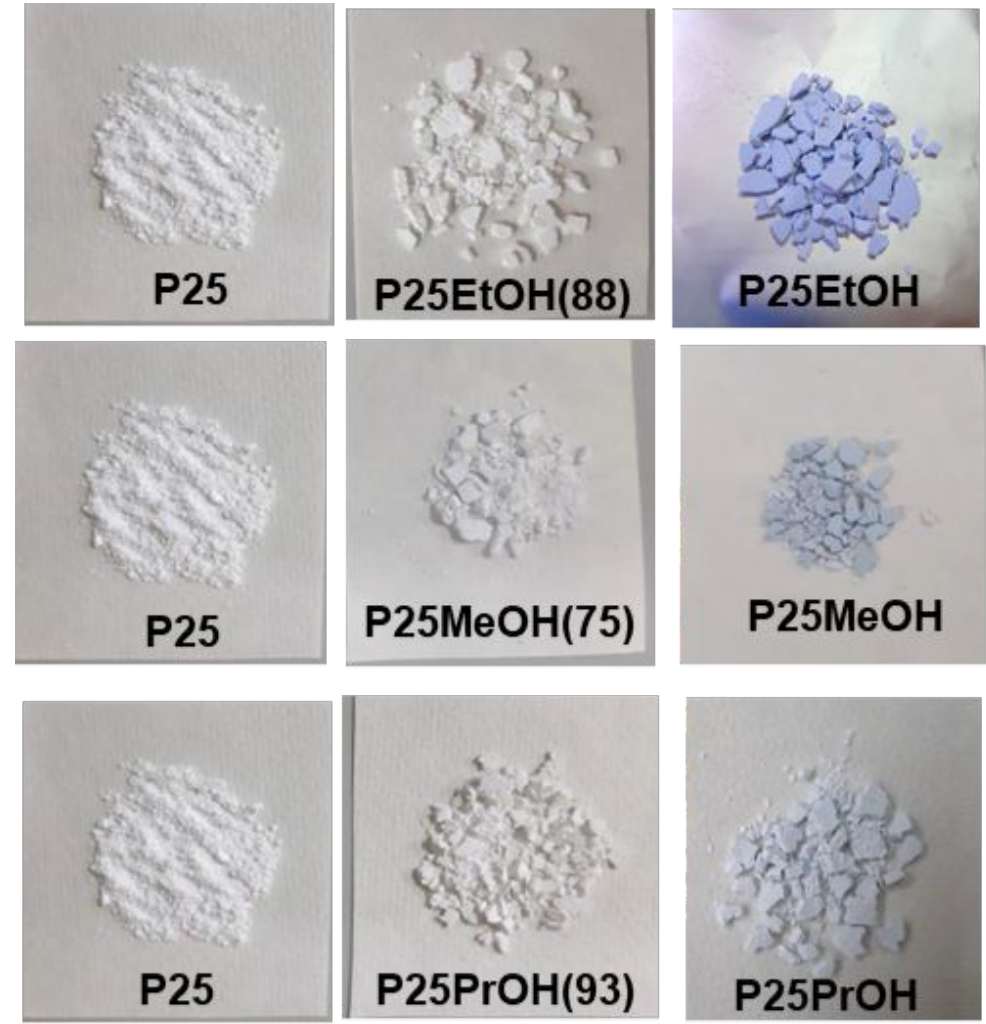

Figure S1. P25 powder before (left column) and immediately after (center and right columns) a 24 hours refluxing treatment in three different alcohols (Top row: ethanol; center row: methanol; bottom row: 2-propanol) and at two different oil bath temperatures: $\mathrm{T}_{1}=\mathrm{T}_{\mathrm{b}}+10^{\circ} \mathrm{C}$ (center column) and $\mathrm{T}_{2}=$ $120^{\circ} \mathrm{C}$. Here $\mathrm{T}_{\mathrm{b}}$ indicates the boiling temperature of the different alcohols. For detail on samples nomenclature see Table 1 in the main text. 

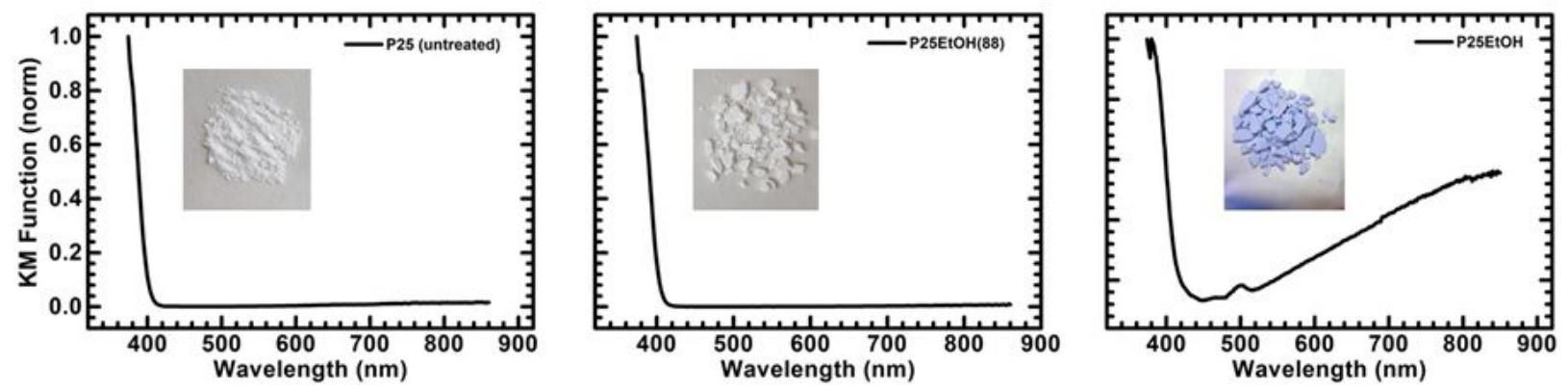

Figure S2. Pictures and peak-normalized experimental Kubelka-Munk curves $F(\lambda)$ for samples P25, P25EtOH(88) and P25EtOH. For detail on samples nomenclature see Table 1 in the main text. 

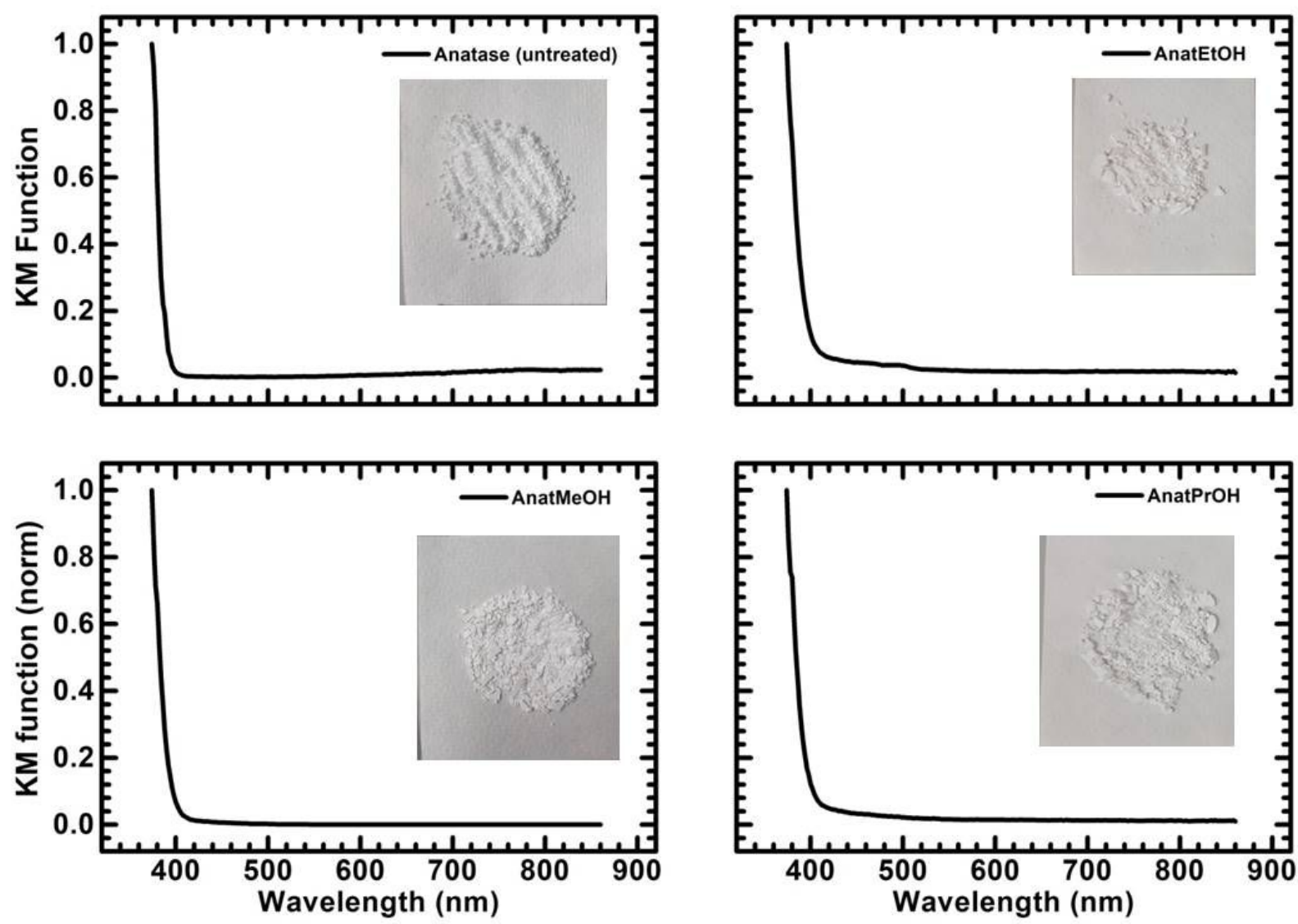

Figure S3. Pictures and peak-normalized experimental Kubelka-Munk curves $F(\lambda)$ for samples Anat, AnatEtOH, AnatMeOH and AnatPrOH. 


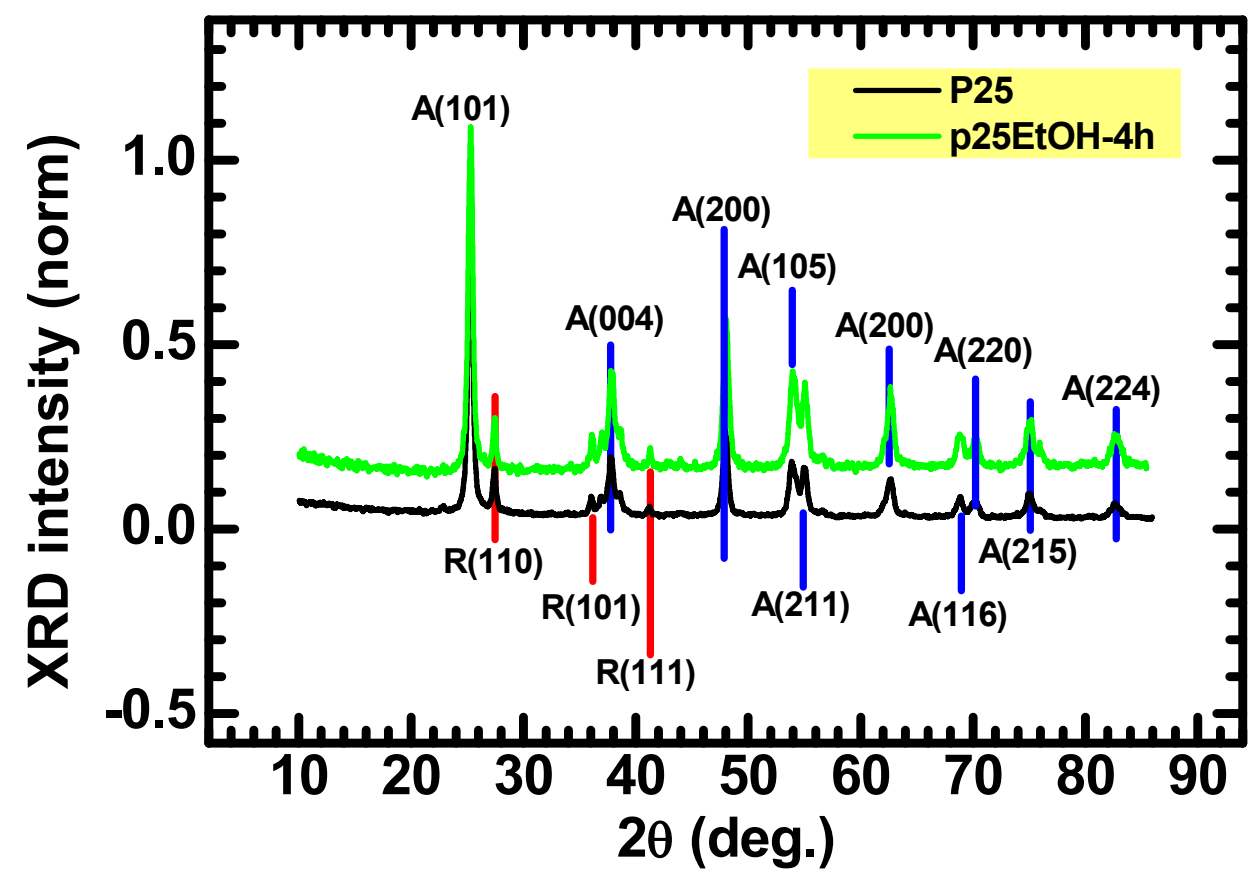

Figure S4. Normalized XRD spectra for pristine and EtOH treated p25 powders (black and green curve, respectively). The diffraction patterns show several contributions originating from the different rutile and anatase planes. No significant differences are evidenced between the samples, indicating that the refluxing treatment in ethanol neither alter the crystalline structure nor causes discernible amount of anatase-to-rutile phase transition. 

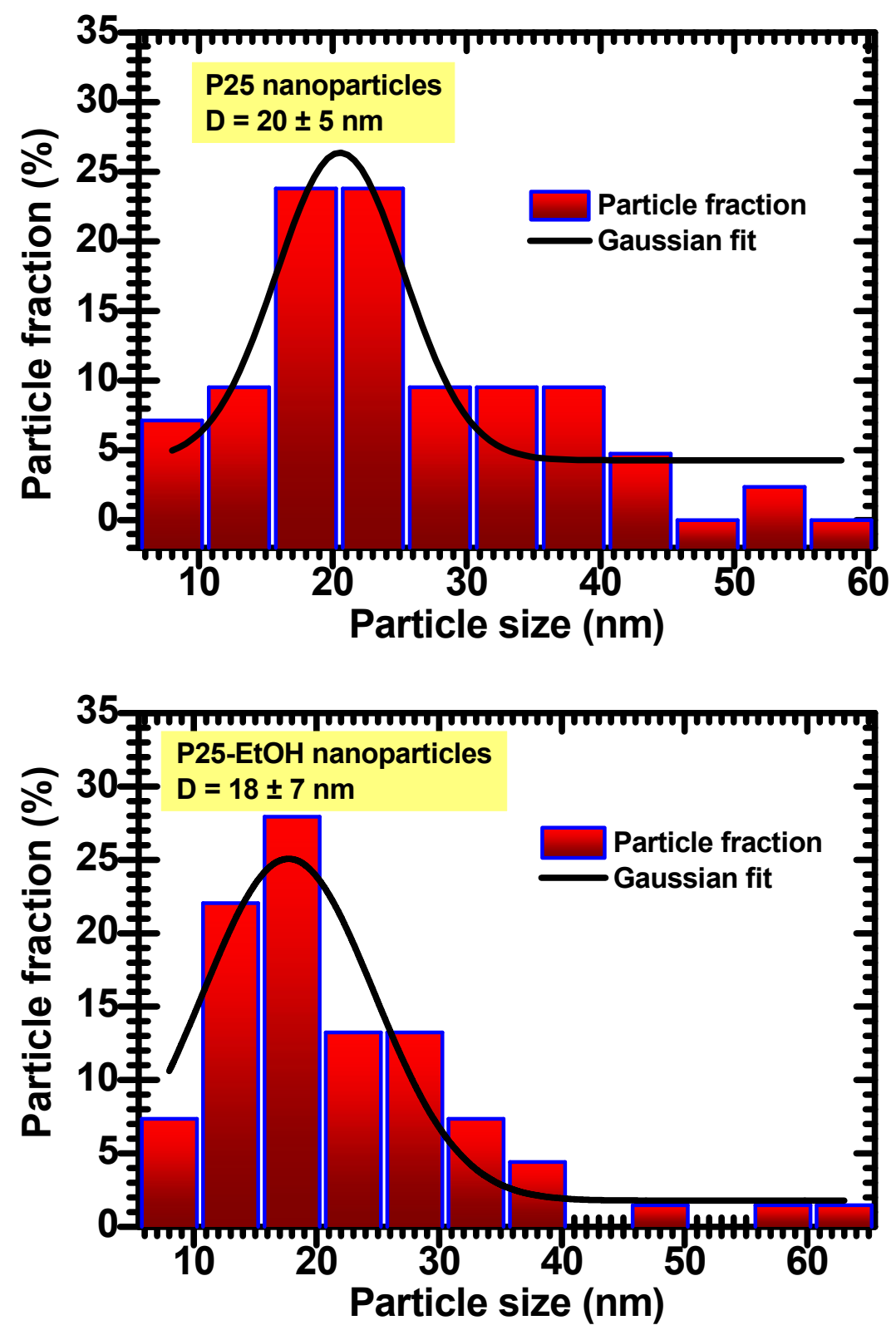

Figure S5. Histogram of particle sizes measured via HRTEM for untreated P25 (top figure) and P25EtOH (bottom figure). The black curve is the best fit curve obtained using a Gaussian distribution. The average and the standard deviation of the size distributions obtained by the Gaussian fits are reported for the quantity D. The number of sampled nanoparticles was $n=42$ for P25 and $n=68$ for P25EtOH. 


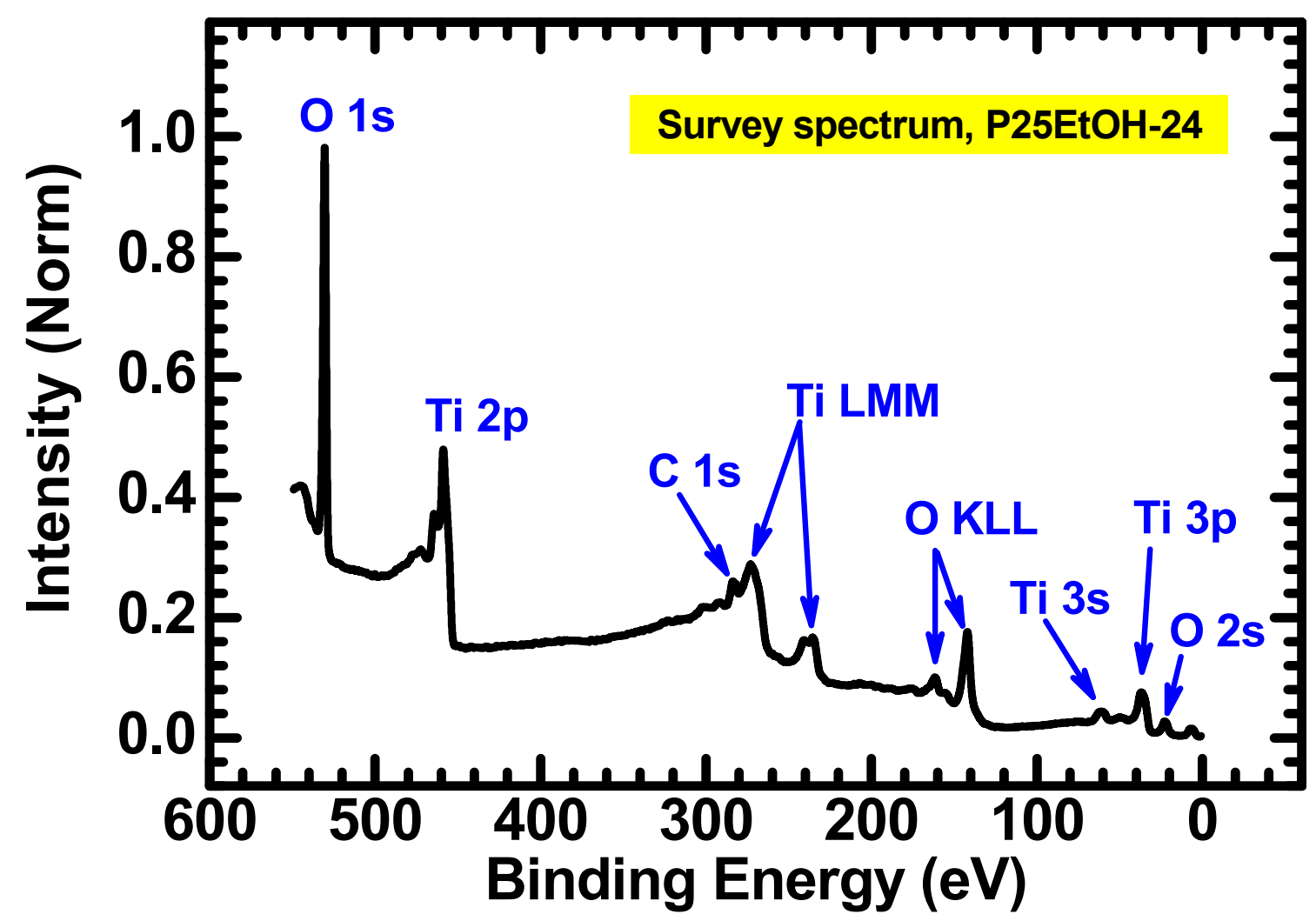

Figure S6. SPEM-XPS survey spectrum for P25EtOH nanoparticles. 

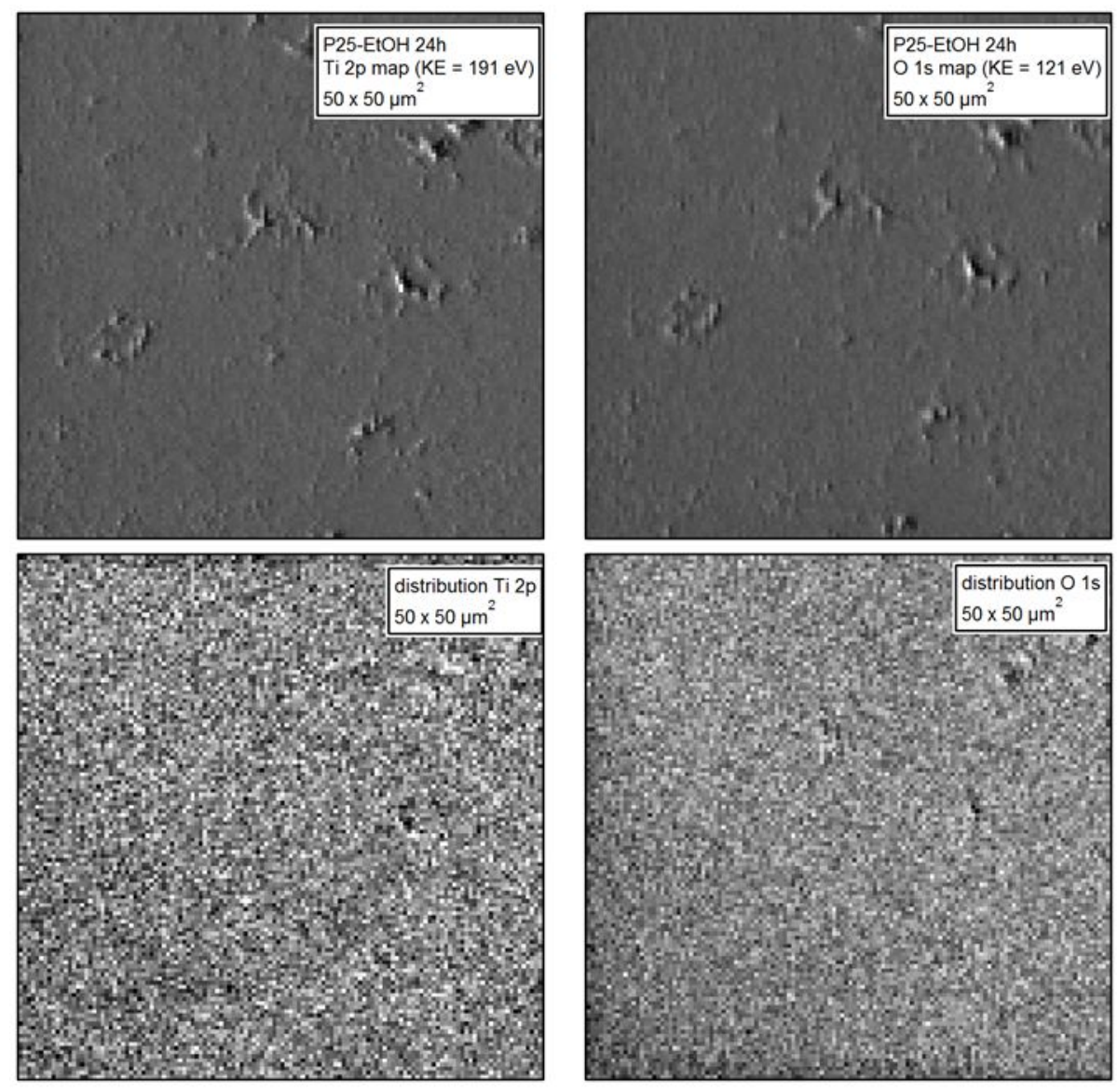

Figure S7. SPEM-XPS microscopy maps on $50 \times 50 \mu \mathrm{m}^{2}$ area imaging the Ti $2 \mathrm{p}$ signal (left) and the $01 \mathrm{~s}$ signal of P25EtOH nanoparticles. The top row shows the raw data which contain a superimposition of chemical and topographical information. In the bottom row the topography was removed (see [L. Gregoratti, A. Barinov, E. Benfatto, G. Cautsro, C. Fava, P. Lacovig, D. Lonza, M. Kiskinova, R. Tommasini, S. Mähl, W. Heichler, 48-Channel electron detector for photoemission spectroscopy and microscopy, Rev. Sci. Instrum. 75 (2004) 64-68. doi:10.1063/1.1630837.] for details) and only the chemical distribution of the elements is shown. The results evidence the spatial homogeneity of the nanoparticles ensemble at micrometer scale, thus justifying the acquisition of XPS spectra in microspot mode with a spot of $70 \mu \mathrm{m}$ reported in the main text. Equivalent results (i.e. powder homogeneity) were obtained also for all of the other investigated samples. 


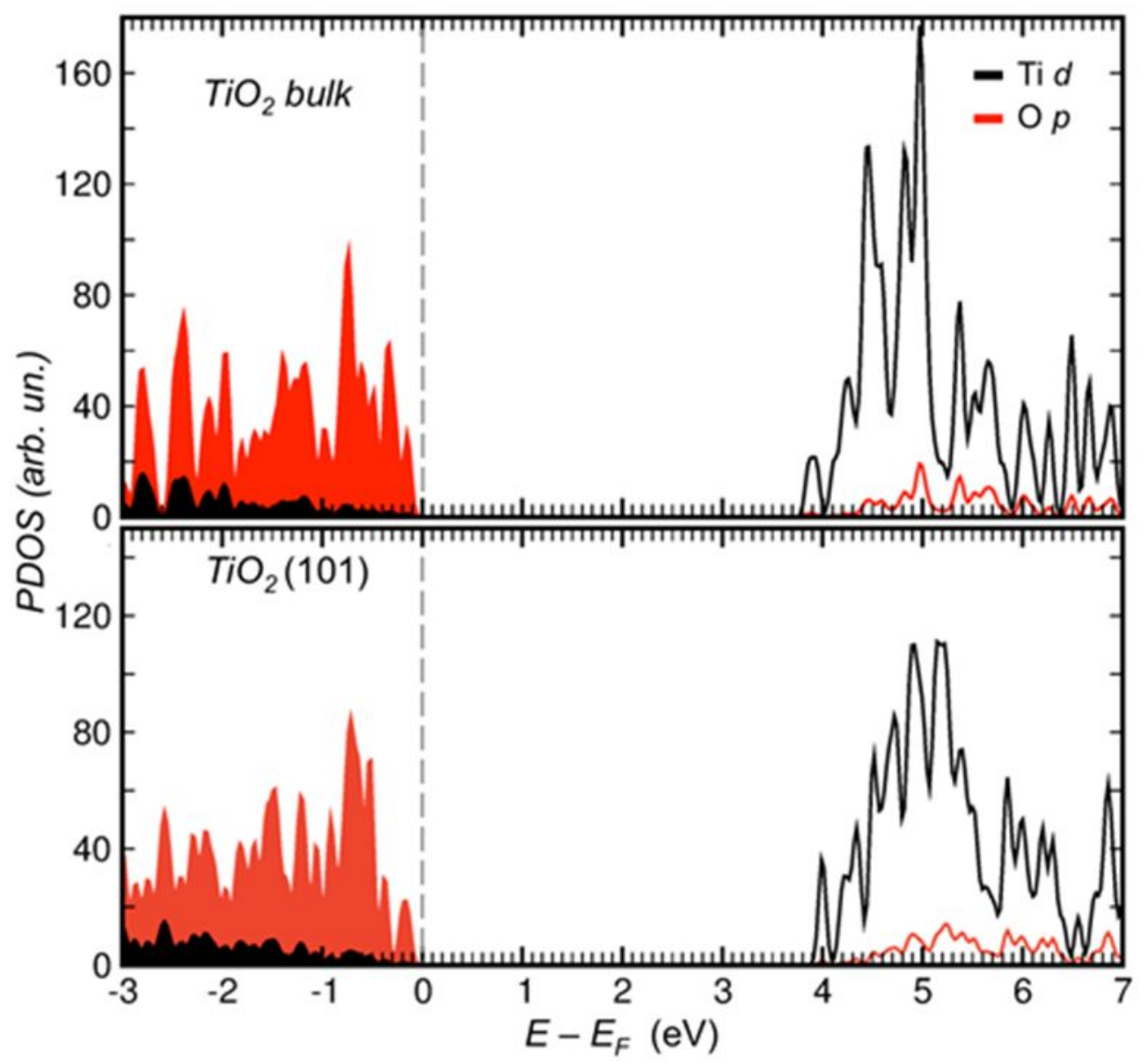

Figure S8. Projected density of states calculated for bulk anatase and for stoichiometric (101) anatase. As in the main article, the black and red lines represent the contributions to DOS derived from Ti $d$ and $0 p$ orbitals, respectively. 


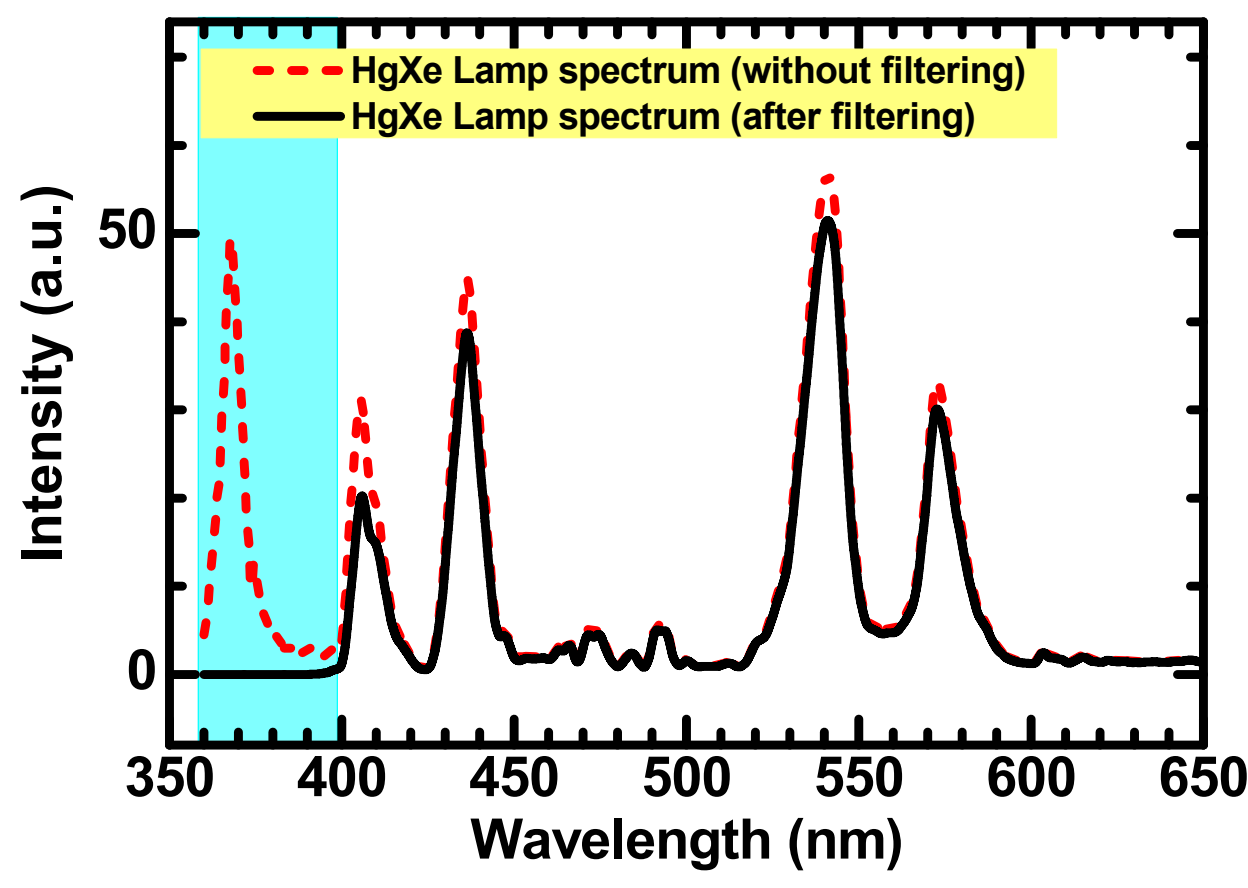

Figure S9. The red dashed curve represents the intensity spectrum of the unfiltered Hg-Xe lamp. The black solid curve represents the intensity of the same lamp after cutting out via optical filters its ultraviolet emission (i.e. the spectral interval evidenced in the cyan-highlighted region). The black curve thus represents the light spectrum actually used in the photocatalytic experiments. 


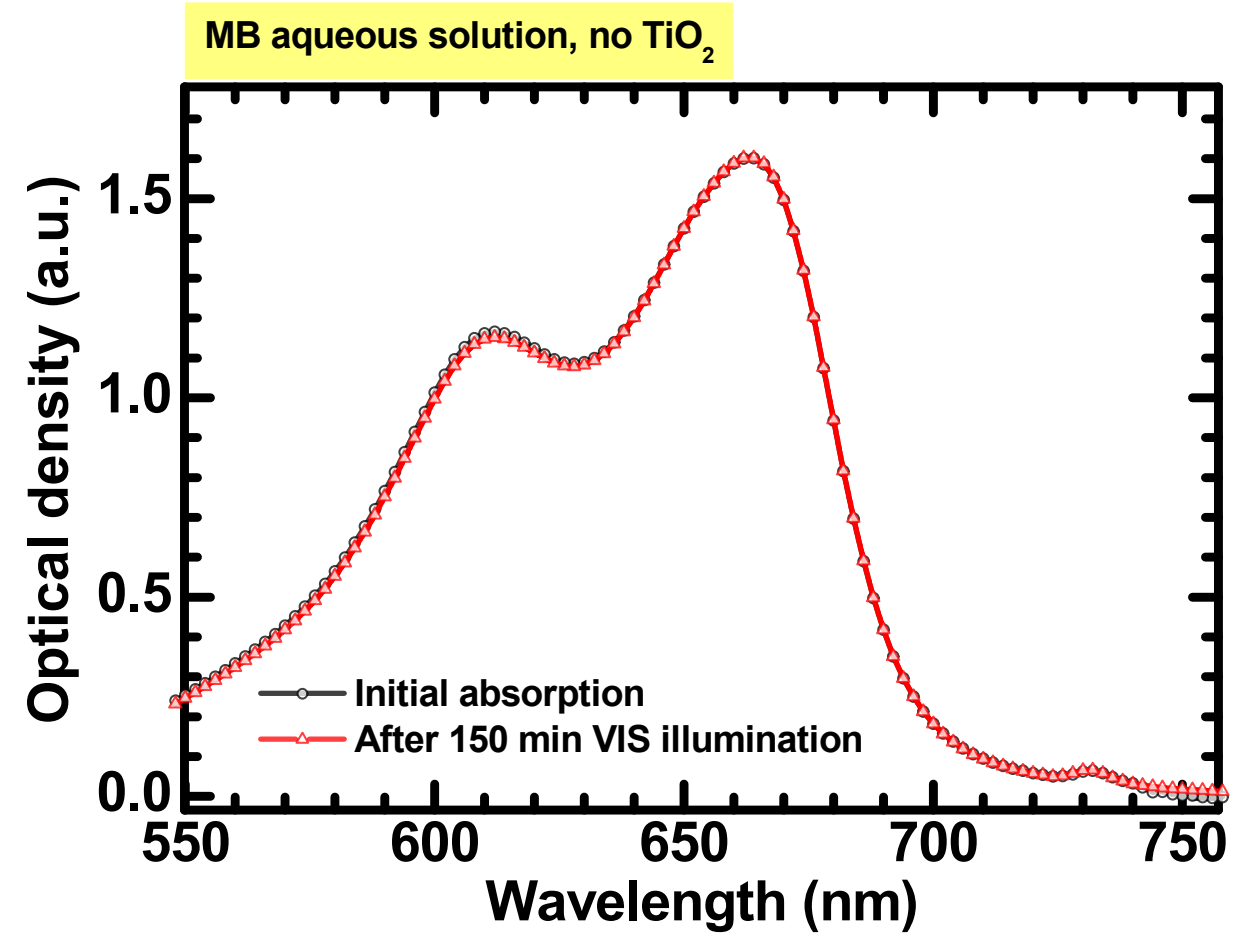

Figure S10. Absorption spectra (optical density) of $\mathrm{MB}$ aqueous solution in absence of $\mathrm{TiO}_{2}$ photocatalyst before (circles) and after (triangles) VIS illumination (150 min). No optical absorption change is observed, indicating the absence of eventual contribution to MB photo-induced decoloration due to photolysis of the MB molecules. 